Página inicial: 534 - Página Final: 544

Tipo de artículo: Investigación

\title{
Experiencia del proceso metodológico para el diseño de planes locales de igualdad de oportunidades en Santander, Colombia
}

\author{
Experience of the Methodological Process for the Design of Equal Opportunity Local \\ Plans in the State of Santander, Colombia
}

Recibido: abril de 2017 Revisado: mayo de $2017 \quad$ Aceptado:junio de 2017

${ }^{1}$ Trabajadora Social, PhD.

Investigaciones Feministas y género. Profesora

Universidad Industrial de

Santander. Contacto:

ahernanq@uis.edu.co

ORCID:

http://orcid.org/0000-0003-

$\underline{1955-0566}$

${ }^{2}$ Historiadora, Msc en Políticas Públicas y Género.

Profesora Universidad

Industrial de Santander.

Contacto:

laurabadillo96@gmail.com

ORCID:

http://orcid.org/0000-0003-

$\underline{3755-7307}$

${ }^{3}$ Trabajadora Social, $\mathrm{PhD}(\mathrm{c})$ en Ciencias Sociales.

Profesora Universidad Industrial de Santander.

Contacto:

rmendezv@uis.edu.co

ORCID:

http://orcid.org/0000-00031005-5809
Por: Andrea Hernández Quirama ${ }^{1}$, Laura Inés Badillo Ramírez ${ }^{2}$ \&

Raquel Méndez Villamizar ${ }^{3}$

\begin{abstract}
In 2015, the territorialization of the public policy on women and gender equality was held in 18 municipalities in the State of Santander, Colombia. It aimed at bringing political decisions agreed by the state government to its municipalities, those related to women's human rights protection and the achievement of gender equity as part of public management. The goal of this article is to present the methodological process carried out for the design of local equal opportunities plans.
\end{abstract}

Key words: Public Policy; Gender Equity; Methodology. 


\section{Introducción}

En Colombia existe la Política Pública Nacional de Equidad de Género para las Mujeres (LPPNEGM) y los Lineamientos del Plan Integral para Garantizar a las Mujeres una Vida Libre de Violencias 2012-2022 (LPIGVLV). Estas políticas buscan incidir en cerrar la brecha entre el reconocimiento y ejercicio de derechos para la mayoría de las mujeres en Colombia; mediante la implementación de un conjunto de acciones estratégicas que aporten en la superación de la discriminación, garantizando el ejercicio de los derechos de las mujeres y con ello beneficiando a toda la comunidad colombiana en pro de una sociedad más equitativa e incluyente (Palacio, 2012).

Durante el periodo 2007-2014 se llevó a cabo el proceso de formulación e implementación de la Política Pública de Mujer y Equidad de Géneros en el departamento de Santander PPMYGES, adoptada por la ordenanza 028 de 2010 (Asamblea Departamental de Santander, 2008).

Este proceso incluyó tres momentos principales: primero, organización e incidencia por parte del movimiento social de mujeres. Segundo, diagnóstico de brechas de género y la formulación del Plan Decenal de Igualdad de Oportunidades y finalmente, implementación de la PPMYGES que comprende tanto la institucionalización como sostenibilidad de la misma. Resultado de este proceso se organizó la PPMYGES a partir de ejes los cuales son: a) Educación para el reconocimiento y realización de los derechos de las mujeres; b) Trabajo digno y remuneración equitativa; c) participación política y representación para la autonomía de las mujeres; d) Santander sin violencias contra las mujeres; e) Santander promueve el derecho de las mujeres a la salud sexual y reproductiva con equidad de género; f) Comunicación y cultura que reconozca y revalorice las diversidades para el desarrollo de Santander, y g) Hábitat, seguridad alimentaria y ambiente (Rodríguez, 2015)

Una vez finalizado este proceso, se identifica escaso reconocimiento y uso de herramientas de planeación del desarrollo local con perspectiva de derechos y género en los municipios de Santander, que ha limitado, de una parte, la formulación y adopción de políticas públicas municipales con su correspondiente plan de igualdad de oportunidades PIO dirigidas a cerrar las brechas de género, generar mayores oportunidades para las mujeres y promover la equidad y, de otra, ha restringido una adecuada territorialización de la PPMYGES.

Territorializar una política pública es una forma de gestión de una política, es llevar al municipio las decisiones políticas pactadas por el gobierno departamental, en este caso las relacionadas con la protección de los derechos humanos de las mujeres y al logro de la equidad entre los géneros. Dicho de otra manera, es aterrizar, bajar a lo local el Plan Decenal de Igualdad de Oportunidades (2010). De esta forma, se lleva a cabo uno de los desafíos de las políticas contemporáneas orientadas a disminuir también las brechas entre los centros y las regiones, lo rural y urbano y en definitiva, para aproximarse a la investigación e intervención social desde una espacialidad o una territorialidad dada. (Gorestein, 2015)

Esto requiere del impulso de procesos de articulación y asistencia técnica que facilite: (a) La implicación del gobierno local en el proceso de la política pública, y (b) La percepción territorializada de los problemas, lo cual se puede traducir en ¿Cuáles son las problemáticas que en términos de desigualdades de género se viven en los municipios? ¿Qué requiere el departamento para dar respuesta y más específicamente la Dirección de Mujer y Equidad de Géneros? - La Dirección de Mujer y 
Equidad de Géneros es la instancia que coordina la implementación de la PPMEG, se encuentra adscrita a la Secretaria de Desarrollo Social de la Gobernación de Santander -.

En este sentido tendrá en cuenta dirigir recursos financieros y técnicos para que se formulen las políticas públicas de equidad de género desde el ámbito municipal, con sus respectivos diagnósticos de brechas de género, planes de igualdad local, y se oriente en relación a la institucionalización de las mismas a través de los acuerdos municipales que las reglamenten.

Con el inició el proceso de territorialización en Santander de la PPMYEG en el 2015, de los 87 municipios del departamento sólo 9 habían adelantado el proceso de formulación de políticas públicas de Mujer y Equidad de Género - Los municipios que habían realizado políticas públicas de mujer y equidad de género en el departamento de Santander antes del proceso de territorialización fueron Bucaramanga, Floridablanca, Piedecuesta, San Gil, Barrancabermeja, California, Socorro, Barbosa y Oiba -.

El objetivo del presente artículo es presentar el proceso metodológico llevado a cabo para el diseño de planes locales de igualdad de oportunidades en 18 municipios del departamento de Santander. En concordancia con los ejes de la PPMYEG anteriormente mencionados.

Se parte de la premisa que las políticas públicas se orientan hacia objetivos comunes para la sociedad, de temas que representan sus necesidades e intereses cuya intencionalidad y causalidad han sido definidas por la interlocución que ha tenido lugar entre el gobierno y sectores de la ciudadanía (Aguilar, 2007). En otras palabras, son decisiones tomadas por actores públicos a fin de resolver un problema puntual de la sociedad. La incorporación en las políticas públicas del tema de equidad de género, surge de un largo proceso político y social que se desarrolla en diversos escenarios con distintos actores, e intereses (Macías \& Rodríguez, 2012)

Por el proceso de los movimientos feministas y de mujeres, la igualdad de género se ha convertido en objeto de responsabilidad pública. Lo que ha promovido la implementación de políticas públicas de igualdad y desarrollo de programas específicos, lo que se constituye en iniciativas de igualdad (Aljama, 2015). En definitiva, varias investigadoras reconocen que existen políticas públicas con equidad de género gracias a que estas organizaciones de mujeres y feministas se han configurado en sujetos sociales e impulsado las problemáticas más apremiantes en la agenda institucional (Araujo, Guzmán, \& Mauro, 2000).

En el caso específico de las políticas de equidad de género, tienen por finalidad fortalecer la ciudadanía y los derechos de las mujeres, para evitar que las desventajas ligadas al hecho de ser mujer, que se generan desde el ámbito de la familia, se extiendan, amplíen o perpetúen a otras esferas como: la educación, el ingreso, los derechos, los cargos profesionales, el poder político, el prestigio y los reconocimientos (Inchaustegui \& Ugalde, 2004). Estas desarrollan medidas con el objetivo de eliminar las discriminaciones por razón de las construcciones de género que limitan las oportunidades de hombres y mujeres para tener acceso y desarrollarse en igualdad de condiciones en diversos ámbitos: político, social, económico y cultural, entre otros (Camberos, 2011).

Estas políticas públicas se encuentran ligadas a distintos conceptos que fueron derroteros fundamentales para la implementación de la metodología en la territorialización de la PPMYGE, cuyo concepto de equidad de género está orientado a: 
“(...) lograr la igualdad de derechos, responsabilidades y oportunidades para mujeres, hombres, niñas y niños. La igualdad no significa que mujeres y hombres serán idénticos, sino que los derechos, responsabilidades y oportunidades de mujeres y de hombres no dependen de si nacen hombres o mujeres. La equidad de género implica que los intereses, necesidades y prioridades tanto de mujeres como de hombres serán tomados en cuenta reconociendo la diversidad de los diferentes grupos de mujeres y hombres. La equidad de género no es un "asunto de mujeres" sino que debe ser objeto de atención y comprometer tanto a hombres como a mujeres. La igualdad entre mujeres y hombres se considera como cuestión de derechos humanos y como una precondición e indicador del desarrollo sostenible centrado en las personas" (Gobernación de Santander, 2010)

Por brecha de género se entiende que "en la mayoría de las sociedades, las mujeres sufren desventajas sociales y económicas debido a la diferencia en la valoración de lo que supone "masculino". Esos diferenciales en el acceso, participación, acceso y control de recursos, servicios, oportunidades y beneficios del desarrollo se conocen como brechas de género. El análisis de las brechas de género permite ver el alcance de las desigualdades en todos los ámbitos" (Secretaria Técnica del Proyecto Equal, 2007)

Los anteriores conceptos fueron la principal malla interpretativa para la construcción metodológica hacia la territorialización de políticas públicas de equidad de género en los municipios priorizados en el departamento de Santander.

\section{Metodología para la territorialización de la política pública de mujer y equidad de género en Santander}

Existen diversos enfoques para abordar los problemas relacionados con la desigualdad de género que fueron desarrollados desde 1960 hasta la actualidad, los cuales son (I) igualdad del trato; (II) igualdad de oportunidades; (III) transversalidad de género; y (IV) paridad (Inchaustegui \& Ugalde, 2004). Estos enfoques describen distintas comprensiones para atender la problemática y generan propuestas encaminadas a promover la equidad de género.

No obstante, las políticas públicas y especialmente las orientadas a la equidad de género son pocos los estudios que describen las metodologías que se llevaron a cabo para la formulación, incorporación y territorialización de las políticas públicas. En parte, se debe a que evaluaciones de políticas públicas de estas características se centran en el impacto y los resultados y poco se describe el proceso que ha sido desarrollado para obtener los resultados de avances o retos en materia de igualdad de género. Por ello, uno de los objetivos en este acápite es describir la metodología y el proceso implementado en la territorialización de la PPMEG en Santander.

Debido a las características y a la magnitud de la terriotirialización de la PPMEG, se distribuyeron los 18 municipios por provincias como se describe en la tabla a continuación: 
Tabla No. 1. Organización por provincias y municipios del departamento de Santander.

\begin{tabular}{|l|l|}
\hline \multicolumn{1}{c}{ Provincia } & \multicolumn{1}{c|}{ Municipios } \\
\hline Provincia Comunera & Socorro, Simacota, Palmas del Socorro, Onzaga \\
\hline Provincia de Vélez & Vélez, Barbosa, La Belleza, Suaita \\
\hline Provincia de García Rovira & San Miguel, Concepción, San Andrés, Carcasí, Guaca \\
\hline Provincia de Mares & $\begin{array}{l}\text { Lebrija, Santa Helena del Opón, Cimitarra, Puerto Parra, San } \\
\text { Vicente de Chucuri. }\end{array}$ \\
\hline
\end{tabular}

Fuente: Elaboración propia

Para iniciar el proceso de territorialización de la PPMEG un primer paso metodológico del proceso fue la organización un equipo de trabajo con idoneidad en los temas de género en distintas áreas, experiencia profesional y capacidades técnicas distribuido de la siguiente manera: Equipo de auxiliares, proveían insumos para el componente cuantitativo con revisión de fuente secundaria por cada brecha priorizada basados en los ejes de la PPMEG y el municipio establecido. Equipo de analistas cuantitativas, con las cuales se establecieron los indicadores y análisis por cada eje priorizado para la elaboración del diagnóstico de brechas de género. Equipo de coordinadoras provinciales y técnicas locales para la planeación de las actividades a realizar en los talleres investigativos y aportes al diagnóstico de los municipios desde lo cualitativo para así elaborar participativamente los documentos de los planes de igualdad por cada municipio. En los talleres participaron mujeres lideresas de las comunidades desde un enfoque diferencial, organizaciones de mujeres y organizaciones sociales y funcionarios y funcionarias de cada una de las dependencias de las alcaldías e instituciones descentralizadas.

Una vez se conformó el equipo de trabajo se procedió con la realización de Mapeos de las instituciones, organizaciones de mujeres y organizaciones sociales, para lo cual se diseñó un formato orientativo que describiera la oferta institucional y las organizaciones presentes en cada una de los municipios. Paralelamente a la construcción de los mapeos, se diseñó una ruta metodológica participativa, incluyente y contextualizada mediante los talleres investigativos para la territorialización de la PPMEG. Estos talleres investigativos se diseñaron para ser una herramienta metodológica que permitía identificar las principales brechas de género y las propuestas para remediar estas problemáticas de manera que fueran priorizadas de acuerdo a la institucionalidad presente y encaminadas hacia la entrega de los modelos de acuerdos municipales y Planes de Igualdad de Oportunidades (PIO) en el marco de la territorialización de la PPMYGE del departamento de Santander.

El primer taller tenía como propósito socializar la PPMYGE e identificar las principales brechas de género por cada uno de los ejes (educación, trabajo, participación política de las mujeres, violencias contra las mujeres, salud sexual y reproductiva, comunicación, cultura, nuevas tecnologías y deporte, hábitat, seguridad alimentaria y ambiente). Se realizó un momento inicial de ambientación donde por medio de ayudas visuales se expusieron los ejes de la política pública en el espacio a trabajar; luego se presentó el proceso y sus alcances, recordando el antecedente de la PPMYGE en Santander y qué es una brecha de género. Posteriormente, se llevó a cabo trabajo por grupos, se revisó y complementó el informe del equipo cuantitativo con las brechas de género identificadas desde lo cualitativo para finalizar el taller con la construcción de acuerdos y la evaluación de la jornada. 
En el segundo taller investigativo en grupos se corroboraron y/o ajustaron las brechas de género y se establecieron propuestas para cada uno de los ejes establecidas en el primer taller investigativo y se recolectaron insumos para la elaboración del Plan de Igualdad y Oportunidades -PIO traducido en propuestas de acuerdo al mapeo y al conocimiento de las personas que participaron, las competencias institucionales y la responsabilidad de otros actores en el marco de los procesos de gobernanza.

Por último, en el tercer taller se validaron las propuestas que conformarían el PIO, las cuales surgieron en el primer y segundo taller investigativo, cuyo propósito era socializar y validar el mismo con la participación de actores sociales e institucionales de cada uno de los municipios. Entre los actores propuestos fueron los Concejos Municipales con el fin de avanzar con la elaboración de los acuerdos municipales a través de la entrega de un modelo de acuerdo. De esta manera el desarrollo del taller tuvo los siguientes momentos, de manera preliminar se realizó la instalación de la mesa principal y acto protocolario, se presentó el PIO, se llevó a cabo la validación teniendo en cuenta la viabilidad de las propuestas de acuerdo a la oferta institucional, y se establecieron acuerdos y compromisos por parte de cada actor municipal. (Ver gráfico 1).

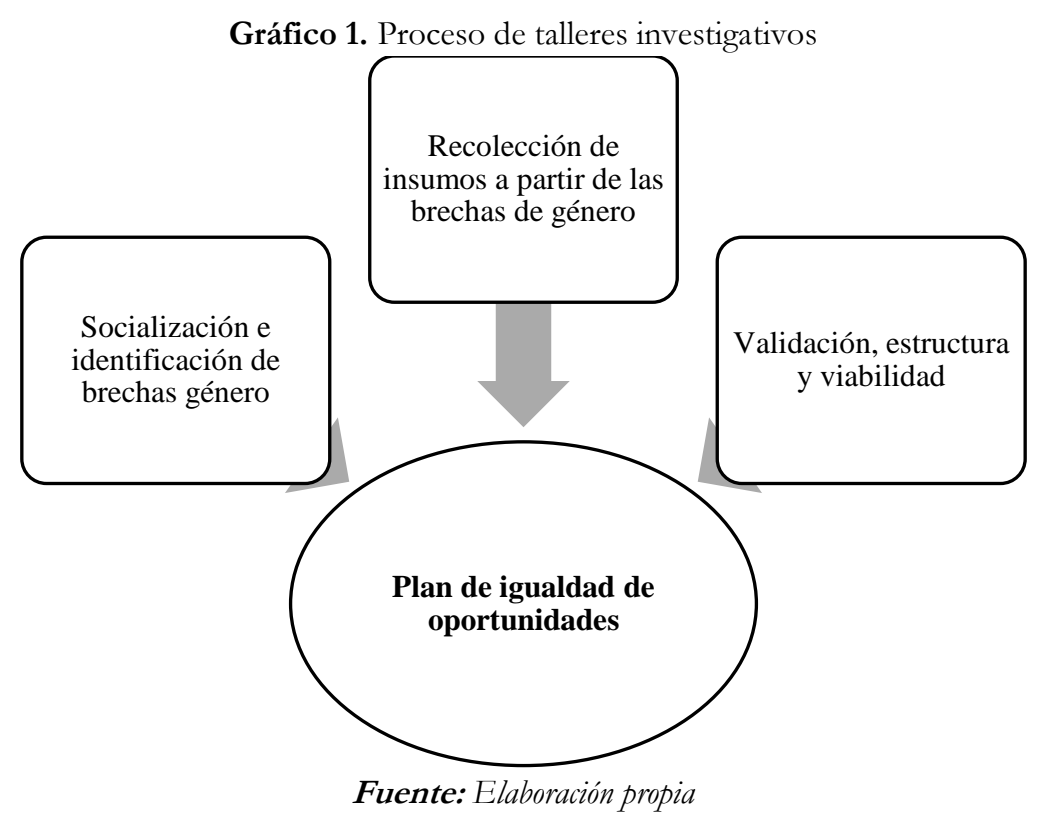

Este proceso metodológico buscaba que las herramientas desarrolladas como los talleres investigativos podrían ser adoptados y ajustados por otros municipios para formular políticas públicas de estas características y/o reforzar las políticas públicas promulgadas que tienen en cuenta la presencia activa de las organizaciones de mujeres, lideresas e institucionalidad.

Para el diseño metodológico de los talleres investigativos, se estableció una ruta pedagógica para fortalecer el proceso participativo y orientar a los equipos de trabajo que emprendían este proceso (Ver gráfico 2). 
Gráfico 2. Ruta pedagógica de los talleres investigativos

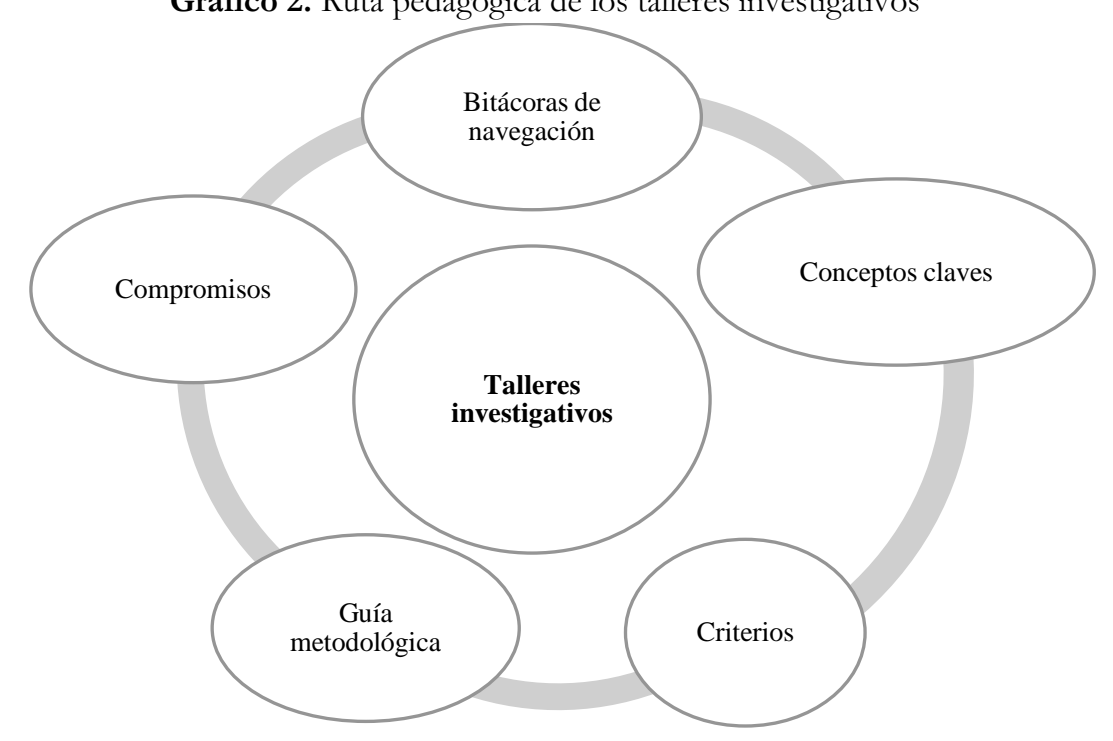

Fuente: Elaboración propia.

Primera fase, las bitácoras de navegación, fueron los documentos relevantes e indispensables para el desarrollo de los talleres investigativos entre los que se encontraban Lineamientos de la Política Pública Nacional de Equidad de Género para las Mujeres (Presidencia de la República de Colombia, 2012), Política Pública de Mujer y Equidad de Género en Santander (Gobernación de Santander, 2010), documento de brechas de género por cada municipio priorizado. Estos documentos se constituyeron en derroteros para demarcar el proceso metodológico en los ámbitos legal y contextual.

Segunda fase, se establecieron conceptos claves, que proporcionaron pistas para realizar los talleres investigativos permitiendo que las y los participantes dispusieran de información que contribuyera a fortalecer su argumentación.

Tercera fase, para la realización de los talleres se establecieron unos criterios que guiaron el proceso metodológico de desarrollo de los mismos, fueron construidos basados en el contexto, las capacidades de los municipios y las organizaciones de mujeres presentes.

Gráfico 3. Criterios metodológicos para los talleres investigativos

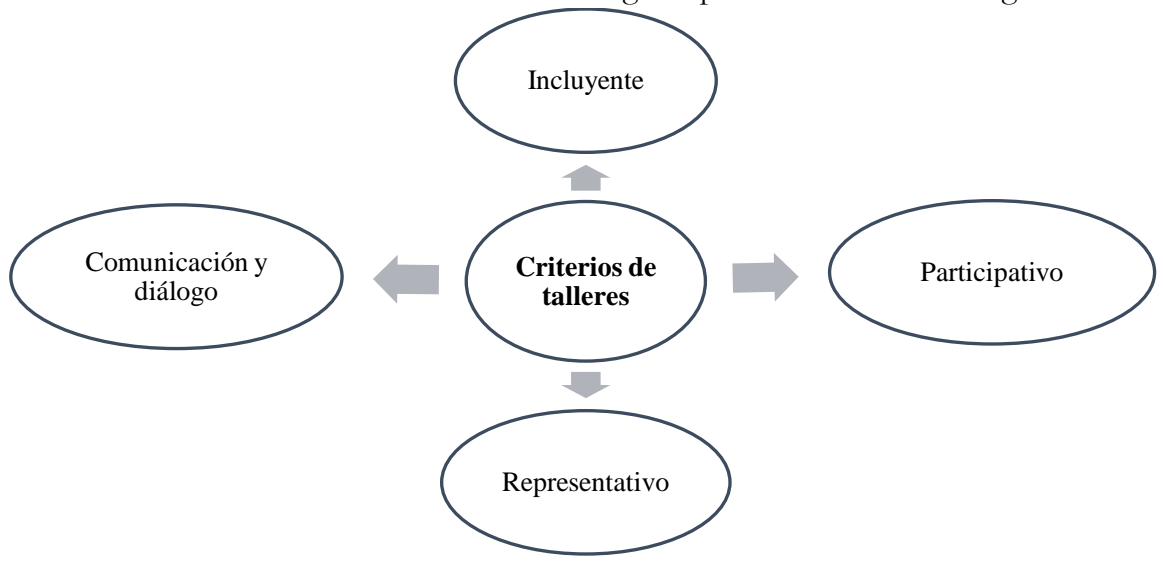

Fuente: Elaboración propia. 
Cada criterio comprendió unas acciones, que se tuvieron en cuenta durante todo el proyecto.

- Incluyente: consideró involucrar a la institucionalidad y a las organizaciones de mujeres y/o lideresas en el proceso. Las políticas públicas necesitan de tomadores de decisiones y a las principales actoras para la veeduría e incidencia de las políticas públicas. De su forma de involucrarse y cómo participan y se integran en los talleres se constituye en la base para el fortalecimiento en la formulación de la política pública y de los PIO. Para ello, se propuso involucrar durante todo el proceso a por lo menos dos instituciones y tres organizaciones o lideresas como hacedores del proceso.

- Participativo: Se procura generar un proceso que permita la participación de diferentes actores y actoras. Brindar herramientas escritas y lúdicas para permitir la circulación de ideas para la identificación de brechas de género y propuestas en el marco de la política pública en los talleres investigativos. Por ejemplo, se elaboraron buzones ubicados en lugares representativos del municipio, que animaron la discusión y generaron propuestas.

- Representativo: Significaba que mujeres y niñas de diversas edades, identidades, saberes y conocimientos que fueran representativas en el municipio. Mujeres diversas con actividades diversas que pudieran dar cuenta de las principales problemáticas vivenciadas y de las respuestas construidas colectiva o individualmente.

- Comunicación y diálogo: Se mantuvo contacto permanente con todas las personas involucradas para dar cuenta de las principales actividades y de los avances del proceso mediante reuniones, visitas, llamadas y envió de información a través del correo electrónico.

La cuarta fase comprendió el diseño de la guía metodológica, donde se presentó la metodología y los pasos para desarrollar en los talleres investigativos. Esta herramienta describió los recursos humanos y materiales para el adecuado desarrollo de las actividades.

La quinta fase se refirió a los compromisos asumidos por parte de las personas participantes en los talleres y de los tomadores de decisión. Estos compromisos, planteaban asistir a toda la fase del proceso y a coadyuvar a incidir para la materialización en los acuerdos expedidos por los concejos de los municipios.

\section{Resultados del proceso metodológico para la territorialización de las Políticas Públicas de Equidad de Género}

Los resultados del proceso metodológico para la territorialización de las PPMYGE se desarrollaron en un periodo relativamente corto donde se articularon diversos saberes y experticias del equipo de trabajo y de la capacidad de las lideresas, de las organizaciones de mujeres en los municipios y organizaciones sociales, quienes aportaron desde su participación con la realización de propuestas.

La metodología planteada permitió obtener los productos como fueron los mapeos, los diagnósticos de las brechas de género y los planes de igualdad de oportunidades - PIO a partir de la realización de 54 talleres investigativos en los municipios priorizados, es decir tres talleres en cada uno de los 18 municipios.

Por tanto, los 18 municipios priorizados cuentan con PIO con objetivos, acciones y responsables por cada uno de los siete ejes de derechos basados en la PPMYGE. Además, se entregaron 18 modelos 
de acuerdo municipal a personas claves de la administración, del Concejo Municipal en el tercer taller investigativo y en reuniones planteadas con las autoridades.

El contar con un equipo de trabajo con capacidades y experiencia en la aplicación del enfoque de género, además con conocimiento de los municipios y reconocimiento en las organizaciones de mujeres, lideresas e institucionalidad en el departamento de Santander, se constituyó en un elemento clave para el desarrollo del proceso.

Por otra parte, iniciar el proceso realizando mapeos de instituciones y organizaciones de mujeres, aportó insumos para tener un panorama institucional de cada municipio, así como una base de datos para utilizar en las convocatorias a los talleres. En términos generales se encontró que algunos municipios contaban con una oferta institucional que contribuía a la implementación de la PPMYGE. Asimismo, que algunos municipios compartían la oferta institucional.

En cuanto a las organizaciones de mujeres se identificó que en algunos municipios no existen, no se encuentran fortalecidas, o no se dedican exclusivamente a exigir la garantía de sus derechos; sino que se encuentran lideresas que poseen una representatividad en el tema.

En las políticas públicas para la formulación, seguimiento de la implementación, veeduría y evaluación es necesaria la configuración de actores que den cuenta e incidan en el proceso. Por ello, se realiza la recomendación de fortalecer las organizaciones de mujeres en sus capacidades de incidencia, organización administrativa, técnica, social y política para que las políticas públicas de mujer y equidad de género se mantengan en el tiempo y puedan contribuir a la superación de las desigualdades y brechas de género que viven especialmente, las mujeres en relación a los hombres en los municipios.

Pese a la permanente convocatoria realizada a las administraciones locales y los Concejos Municipales, estas no se articularon en su totalidad en el marco del proceso de territorialización de la política pública de mujer y equidad de género en los municipios. Lo cual, puede incidir en la adopción de los acuerdos municipales. Únicamente se adoptaron dos políticas públicas de mujer y equidad de género en dos municipios (San Miguel \& Onzaga).

Según (Palacio, 2012), la política requiere de una voluntad social para emprender o continuar el proceso de inserción de la perspectiva de género en todas las instituciones, para lo cual es imprescindible el compromiso de los/as gobernantes, de los niveles directivos y administrativos y de los/as operadores/as jurídico/as y de las demás fuerzas sociales, en especial de la academia.

Finalmente, el proceso de territorialización permitió evidenciar que existe una institucionalidad, organizaciones de mujeres y lideresas del área urbana y rural con poca información y conocimientos en los temas de equidad de género. Lo cual se constituye en obstáculo para el desarrollo del ejercicio de los derechos de las mujeres, por lo tanto se requiere continuidad en las acciones de capacitación, formación y acompañamiento institucional para fortalecer los procesos organizativos, para así incidir en la integración de las mujeres a los procesos de desarrollo y participación política (Palacio, 2012).

\section{Conclusiones}

Para iniciar es necesario mencionar, que la búsqueda de información y recolección de datos para identificar las brechas de género, fue un proceso dispendioso, dado que la revisión de bases de datos, 
la información no se encuentra desagregada por sexo, edad, área rural y urbana, ni menos sobre todos los ejes trabajados en la PPMEYG. Lo que evidencia la ausencia de información, en este sentido, urge la necesidad de establecer mecanismos en el país y en la región que suministre información fidedigna y unificada sobre la situación y condición de las mujeres en diferentes áreas.

El diseñar la metodología con el equipo de trabajo que estaba en las regiones, permitió la apropiación del proceso en su implementación y seguimiento; asimismo, que el equipo fuera de las regiones, aportó elementos valiosos, en especial el conocimiento de la zona, la capacidad de convocatoria, la validación y enriquecimiento de los datos cuantitativos de los diagnósticos por brechas de género.

Para lograr implementar los Planes de Igualdad y Oportunidades en los municipios, se requiere voluntad política, donde las administraciones y Concejos Municipales adopten mediante acuerdos municipales dichos Planes, y así poder darle un sustento legal, político y financiero, mediante un plan operativo anual con presupuesto y un ente coordinador de las actividades con poder de decisión y seguimiento, todo acorde a la Política Pública Departamental de Mujer y Equidad de Género.

En este sentido, fortalecer las capacidades de las mujeres en conocimientos de derechos de las mujeres, equidad de género, legislación y veeduría, se hace fundamental para darle continuidad al proceso a corto, mediano y largo plazo con los Consejos Consultivos de Mujeres. Además de visibilizar los avances realizados en eventos públicos donde se invite y participen los alcaldes, alcaldesas, concejos municipales y gobernación de Santander para comprometerlos con el proceso de territorialización al inicio, durante y al finalizar el proceso.

Como aprendizaje derivado de este proceso podemos inferir que es importante diseñar una metodología hacia la incidencia política de los tomadores de decisión con el fin que se articulen los procesos de territorialización de la PPMEG, y que estas sean un camino articulado y liderado por las máximas autoridades del departamento. En este sentido, se estaría abonando en la sensibilización y en la priorización de los derechos de las mujeres y de las políticas públicas que los promueven con la participación y compromiso de estos actores políticos y con poder de decisión en la adopción e implementación de políticas públicas orientadas hacia la equidad de género.

\section{Referencias bibliográficas}

Aguilar, L. (2007). Marco para el análisis de las políticas públicas. I Encuentro presencial de la maestría en Políticas Públicas Comparadas. México: FLACSO.

Aljama, E. (2015). Género, poder y administraciones públicas: sobre la (im)posibilidad del cambio hacia una mayor igualdad. Una revisión de la literatura. Revista Española de Ciencia Política, 263287.

Araujo, K., Guzmán, V., \& Mauro, A. (2000). El surgimiento de la violencia doméstica como problema público y objeto de políticas. Revista de la CEPAl, 133-145.

Asamblea Departamental de Santander. (2008). Acuerdo 028 Política pública de mujer y equidad de género de Santander. Bucaramanga. 
Camberos, M. (2011). Empoderamiento femenino y políticas públicas, una perspectiva desde las representaciones sociales de género. Entramado, 40-53.

Gobernación de Santander. (2010). Política pública de Mujer y Equidad de Géneros en Santander. Plan decenal de igualdad de oportunidades de Santander PPMYGES-PIO 2010-209. Bucaramanga.

Gorestein, S. (2015). Transformaciones territoriales contemporáneas. Desafíos del pensamiento latinoamericano. Revista Latinoamericana de Estudios Urbano Regionales, 41-122.

Inchaustegui, T., \& Ugalde, Y. (2004). Materiales y herramientas conceptuales para la transversalidad de género. Observatorio ciudadano de políticas de niñez, adolescencia y familia.

Macías, R., \& Rodríguez, G. (2012). Género: de lo académico a lo político. El Cotidiano, 79-87.

Palacio, Y. (2012). Comentario a la política pública de equidad de género para las mujeres. Vniversitas, 11-14.

Presidencia de la República de Colombia. (2012). Lineamientos de la política pública nacional de equidad de género para las mujeres. Bogotá: Alta Consejería Presidencial para la Equidad de la Mujer.

Rodríguez, J. (2015). El movimiento de mujeres y su incidencia en la formulación e implementación de la política pública de la mujer y equidad de Géneros en Santander, Colombia, 2007-2014. Reflexión política, 162-176.

Secretaria Técnica del Proyecto Equal. (2007). Glosario de términos relacionados con la transversalidad de género. 